\title{
Tuberous sclerosis - A rare presentation in neonate
}

\author{
${ }^{1}$ Tapan Sinha Mahapatra, ${ }^{2}$ Asim Kumar Mallik, ${ }^{3}$ Manik Mondal, \\ ${ }^{4}$ Chinmoy Lath, ${ }^{5}$ Isita Tripathy
}

Department of Pediatric Medicine, Nilratan Sircar Medical College and Hospital, The West Bengal Uuniversity of Health Sciences, India)

\begin{abstract}
Tuberous sclerosis is a neurocutaneous autosomal-dominant disorder, presents with highly variable clinical manifestation and affects multiple organ system. Its manifestation in neonate is poorly described. We report a case of intractable neonatal seizure with multiple hypopigmented patch. Neuroimaging of brain shows classical cortical tubers and subependymal nodule suggestive of tuberous sclerosis in newborn. Hence tuberous sclerosis should be considered as an etiology of neonatal seizure and neuroimaging should be performed when other common causes of neonatal seizure have been excluded.
\end{abstract}

Keywords: Neonatal seizure, Neuroimaging,Tuberous sclerosis

\section{INTRODUCTION}

Tuberous sclerosis is a protean disorder, chiefly manifested by mental deficiency, epilepsy, and skin lesions. It occurs with a frequency of 1 in 6,000 to 9000 and is transmitted as an autosomal dominant gene. Approximately one-third of cases have inherited a mutated TS gene from one of the parents; the rest are new mutations[1]. Abnormalities can be found in the brain, eyes, skin, kidneys, bones, heart and lungs. In the brain, three types of abnormalities occur: cortical tubers, subependymal nodules, and disorders of myelination. The most characteristic gross abnormality is the presence of tubers. These are numerous hard areas of gliotic tissue of varying size, after which this condition is named. Tubers can be located in the convolutions of any part of the cerebral hemispheres.Tubers may be dynamic lesions characterized by populations of cells undergoing proliferation, migration, and death. Tuberous sclerosis complex is a multisystemic neurocutaneous disorder, manifesting variably during infancy and childhood that remains poorly described in neonates[2]. When described in this population, tuberous sclerosis complex is most commonly associated with cardiac rhabdomyomas and brain tumors, but is rarely mentioned as an etiology for neonatal seizures. We report a case of intractable neonatal seizure presenting from day 1 of life which on relevant investigations diagnosed to be a case of TUBEROUS SCLEROSIS.

\section{HEADINGS}

1.Case history: Single, Term $40 \mathrm{wk}$ gestation, $2.8 \mathrm{~kg}$ birth weight, born by normal vaginal delivery on 28th November 2012 at 9 am (IST), cried immediately after birth and was put on breast within 1 hour of delivery. Mother noted few abnormal movement during initial breast feeding (within first hour of life), baby otherwise was feeding well. No history of meconium stained liquor, or foul smelling liquor, premature rupture of membrane or prolonged labour, nor any history of significant maternal fever, skin rash, neck swelling or any genital lessions during the course of pregnancy. No family history of seizure or any evidence of neurocutaneous marker or of mental retardation in the family was found.

2.Examination: On head to foot examination, No obvious life threatening congenital anomaly noted. Head Circumference $-35 \mathrm{~cm}$; Chest circumference- $29 \mathrm{~cm}$; length- $56 \mathrm{~cm}$; weight $-2.8 \mathrm{~kg}$. Skin examination revealed five distinct hypoigmented patch ( Ash leaf macule) at left leg (calf region), at right buttock region(Fig 1), at back of right thigh, radial aspect of distal right forearm and one at center of forhead. However there are no cafe-a-laspot, shagreen patch, or any adenoma sebaceum.

3.Management: Baby was managed on the line of neonatal seizure. Capillary blood glucose was constantly within normal limit and serum calcium was done thrice which was also within normal limit.Patient recieved phenobarbitone in maximum dose $(40 \mathrm{mg} / \mathrm{kg})$ and phenytoin in max dose $(40 \mathrm{mg} / \mathrm{kg}) \mathrm{I} . \mathrm{V}$ to control seizure and thereafter put on maintenance dose of $5 \mathrm{mg} / \mathrm{kg}$ in two divided doses of each. There was poor control of seizure with these drug and so the patient was give pyridoxine $100 \mathrm{mg}$. Failing to control seizure, patient was put on $\mathrm{mcg} / \mathrm{kg} / \mathrm{min}$ to have adequate control of seizure. After having seizure free for $48 \mathrm{hrs}$, midazolam was started to tapper. Patient developed occasional seizure on withdrawing the dug. Failing to control seizure adeqately, Sodium valproate was added orally, initially $30 \mathrm{mg} / \mathrm{kg} /$ day which was increased to maximum dose of 60 $\mathrm{mg} / \mathrm{kg} / \mathrm{day}$ in three divided dose orally. Patient finally had acceptable seizure control on phenobarbitone (5 
$\mathrm{mg} / \mathrm{kg} /$ day P.O), phenytoin (5 mg/kg/day P.O) and sodium valproate (60 mg/kg/day P.O). However, seizure did not stop completely and patient continue to have few episodes of seizure every day.To exclude other causes of seizure, following investigations were done:Serum electrolyte - Na - $139 \mathrm{mEq} / \mathrm{L} ; \mathrm{K}-2.5 \mathrm{mEq} / \mathrm{L}$; Calcium - 10.2 $\mathrm{mg} / \mathrm{dl}$.Sepsis screen was done which was not very conclusive - Hb 18.5; TC 12000; N39,L54,M2,E5,B0, No toxic granules; platelet- 4,00,000; CRP $1.09 \mathrm{mg} / \mathrm{dl}$. Blood culture shows no growth. CSF study - glucose- 41 $\mathrm{mg} / \mathrm{dl}$, protein $70 \mathrm{mg} / \mathrm{dl}, \mathrm{Cl}-130 \mathrm{mEq} / \mathrm{L}$; Cell count - 35, L95\%, N5\%, Gram stain - no organism found. IEM screening was negative. Screening CT scan was done which revealed Multifocal hyperdensities at B/L paraventricular areas.TORCH serology was performed to exclude congenital TORCH infection and was found to be negative.MRI scan of brain - multiple sub ependymal and cortical tuber in a case of tuberous sclerosis(Fig 2). To find other stigmata of the TSC disease, following work up was done:USG of Abdomen - No renal angiomyolipoma seen nor any other cyst noted at any other abdominal organs. Fundoscopy- No retinal hamartoma noted. Chest xRay - normal study. Echocardiography - Echo reflective well circumscribed scattered mass seen in right ventricle apex and at inter ventricular septum suggestive of cardic rhabdomyoma(Fig 3).

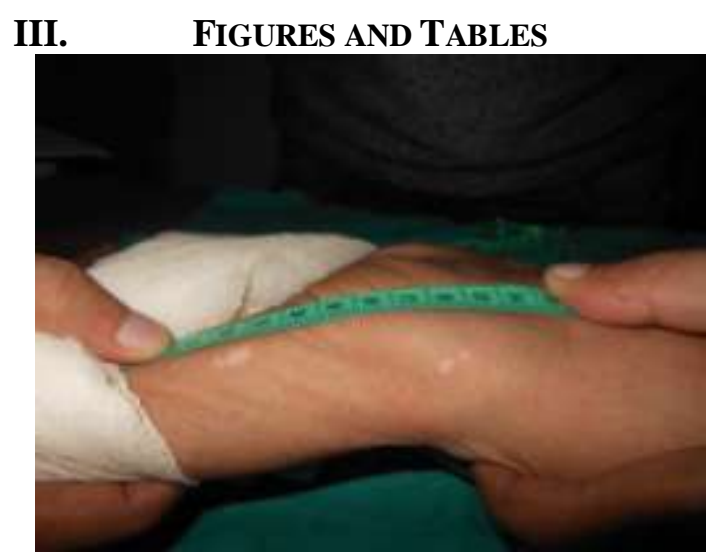

Figure 1:Ash leaf macule

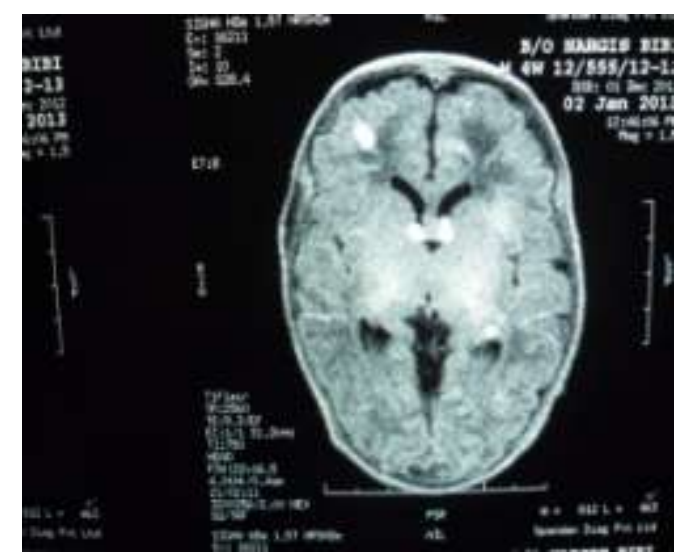

Figure 2: MRI scan showing subepndymal and cortical tubers

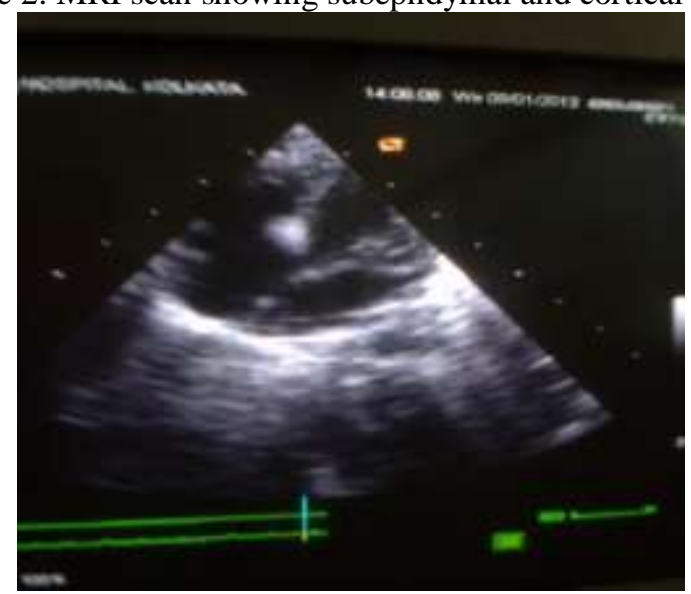

Figure 3: Cardiac Rhabdomyoma 


\section{CONCLUSION}

Major features of TSC are cortical tubers, subependymal nodule, subependymal giat cell astrocytoma, Facial angiofibroma or forhead plaque, ungal or periungal fibroma (non traumatic), hypomelanotic macules $(>3)$ , Shagreen patch, .multiple retinal hamartomas, Cardiac rhabdomyoma, renal angiomyolipoma, pulmonary lymphangioleimyomatosis. Minor features are Cerebral white matter migration lines, multiple dental pits, gingival fibromas, Bone cysts, retinal achromatic patch, confetti skin lesions, non renal hamartomas, multiple renal cysts, and hamartomas rectal polyp.Definite diagnosis of TSC is done if there is 2 major or 1 major and 2 minor fetaures[3]. In our case we find the presence of 4 major features and hence the definite diagnosis of tuberous sclerosis. Respiratory dystress, arrythmia, murmurs and cardiomegally are the main signs initially in the neonates. Cardiac rhabdomyomas comprises $32.8 \%$ of the TSC pathological findings; those of central nervous system origin, $47.5 \%$; and renal cystic disease, $13.2 \%$. Skin lessions and retinal hamartomas were noted rarely at birth[4]. But in our case cutaneous marker of tuberous sclerosis is present which is rarely found in neonates with TSC. The baby presented with intractable neonatal seizure which is also not a common presentation in neonate with tuberous slerosis. hence in respect of this our case is also different .The presence of neurocutaneous stigmata have guided us to search for other evidence in support of tuberous sclerosis. As because neurocutaneous stigmatas are rare in newborn[1], we should go for brain neuroimaging and other investigations for search of tuberous sclerosis keeping in mind the possibility of tuberous sclerosis in neonate presenting with intractable seizure.

\section{REFERENCES}

[1] John H. Menkes, Harvey B. Sarnat, Bernard L. Maria Neurocutaneous syndrome, Child neurology $7^{\text {th }}$ edition,(California, Williams and willkins 2006),

[2] Miller SP, Tasch T, Sylvian M, Farmer JP, O’Gorman AM, Shevell MI. Tuberous sclerosis complex and neonatal seizures. J Child Neurol,13(12), 1998 Dec,619-23

[3] Mustafa Sahin,Tuberous Sclerosis,Nelson Textbook of Pediatrics, $19^{\text {th }}$ edition (India:Elsevier, 2012)2049-2051

[4] Isaacs H, Perintal (fetal and neonatal) tuberous sclerosis: a review, Am J Pernatol.26(10), 2009 Nov,755-60 